\title{
Sleep apnea syndrome and hypothyroidism
}

\author{
Fabio Lanfranco
}

Received: 11 July 2013/Accepted: 12 July 2013/Published online: 12 October 2013

(C) Springer Science+Business Media New York 2013

Obstructive sleep apnea syndrome (OSAS) is a serious, prevalent condition that has significant mortality and morbidity when untreated. It is strongly associated with obesity and is characterized by several endocrine and metabolic abnormalities that are frequently more marked than those in obese patients without OSAS, suggesting that factors other than obesity per se have a role in this clinical condition [6]. Obese patients with OSAS show alterations in the GH/IGF-I axis function, PRL secretion, HPA axis and gonadal axis activity. Some derangement in TSH secretion in obesity with or without OSAS has been demonstrated by some authors, whereas a normal thyroid activity has been described by others $[5,6]$.

The study by Mete and colleagues published on Endocrine in April 2013 examined the relationship between OSAS and thyroid disease. Functional and ultrasonographic examination of the thyroid gland was performed in 150 polysomnographically diagnosed OSAS patients and in 32 control subjects. Aim of the study was to evaluate both thyroid function and thyroid nodule frequency and parenchyma heterogenecity in OSAS patients. The morphologic evaluation of the thyroid gland is peculiar of this study in comparison to other investigations. In fact, regardless of hypothyroidism, large goiters alone can cause pharynx occlusion and thus OSAS. The authors did not find any difference in prevalence of hypothyroidism, numbers of nodules, and parenchyma heterogenecity determined by ultrasound, between OSAS subgroups and controls. Thus this study did not reveal any relationship between OSAS

\section{F. Lanfranco $(\bowtie)$}

Division of Endocrinology, Diabetology and Metabolism, Department of Medical Sciences, University of Turin, Corso Dogliotti 14, 10126 Turin, Italy

e-mail: fabio.lanfranco@unito.it and thyroid disease. The authors concluded that long-term follow-up studies are needed to establish the possible significance of routine evaluation of OSAS patients for thyroid disease [9].

As a matter of fact, the incidence of thyroid deficiency states in patients with OSAS is not clearly known, thus the opportunity to evaluate thyroid function in all patients with the syndrome is still debated.

It is well known that OSAS and hypothyroidism have several common signs that create a significant risk of undiagnosed hypothyroidism in OSAS. On the other hand, the suspect of sleep apnea in hypothyroid patients, especially when obese and with persistent sleep-disordered breathing during appropriate replacement therapy, should be taken into account.

Endocrinologists and professionals dealing with sleep disturbances and OSAS should bear in mind that hypothyroidism may cause OSAS and that OSAS may be linked to (or even cause) hypothyroidism.

A link between hypothyroidism and OSAS is suggested by the high prevalence of sleep apnea among hypothyroid patients, particularly in rare myxedematous cases [3, 7]. The increased prevalence of OSAS appears to be related to obesity and male sex rather than to hypothyroidism per se, although decreased ventilatory responses, extravasation of albumin, and mucopolysaccharides in the tissues of the upper airway and hypothyroid myopathy have been suggested as possible contributing factors for OSAS in hypothyroidism [3]. Moreover, contrasting data are available concerning the efficacy of thyroid replacement therapy in improving sleep apnea in patients with clinical hypothyroidism. Some authors describe a prompt reversal of symptoms, sleep-disordered breathing, and nocturnal hypoxia [12], while little or no improvement in sleep apnea is reported by others [10]. Nocturnal Continuous Positive 
Airway Pressure (nCPAP) treatment can be necessary initially and in some patients even after remission of clinical signs of hypothyroidism [11].

Concerning the role of OSAS in inducing thyroid dysfunction, in a group of obese patients with OSAS I have not found an impaired thyroid activity in basal conditions nor an altered TSH response to TRH challenge test [5], in agreement with some but not other studies. In fact, some derangement in TSH secretion in obesity with or without OSAS has been demonstrated [2, 4]. Bratel et al. [2] have reported a more pronounced reduction of serum TSH in OSAS patients with the most severe nocturnal hypoxemia, with a normal TSH response to TRH before and after nCPAP treatment. In 101 overweight and obese male patients, Meston et al. [8] have described a small significant inverse correlation between OSAS and free T4 levels but not TSH, with no apparent association between obesity and either hormone. One-month nCPAP treatment compared to placebo resulted in a significant reduction in TSH without elevation in free T4 levels, consistent with the pattern of recovery from non-thyroidal illness.

Overall the prevalence of hypothyroidism in patients suspected of having OSAS does not seem to be different from the one in the general population, but it is greater in patients with OSAS. Interestingly, Bahammam et al. [1] showed that the prevalence of newly diagnosed clinical hypothyroidism in OSAS patients is low while subclinical hypothyroidism is common. Mete et al. [9] did not find a significant difference in the prevalence of subclinical and overt hypothyroidism in OSAS patients and controls.

Given, the overlap in clinical presentation of primary OSAS and hypothyroidism, some authors indicate that screening for hypothyroidism is required to prevent misdiagnosis and that it is a cost-effective component of the investigation of sleep apnea [12]. On the other hand, this screening in patients with OSAS is not considered necessary by several authors unless the patient is symptomatic or belongs to a risk group [10].

In conclusion, much remains to be learned about the diagnosis and treatment of sleep apnea syndromes associated with thyroid hormone deficiency, and further investigations are needed. I agree with Mete and colleagues on the importance of considering a possible incidental thyroid dysfunction in patients with OSAS. Missing the diagnosis of hypothyroidism in OSAS patients may lead to inappropriate or unnecessary nCPAP treatment. However, clinicians should be aware that missing the diagnosis of
OSAS (in hypothyroid patients) may lead to an increased morbidity and mortality due to the well-known increased cardio-vascular risk of this syndrome. Thus, all professionals dealing with hypothyroid patients and/or with subjects with sleep disordered breathing should keep in mind the interrelation existing between OSAS and thyroid dysfunction and address selected patients to more specific evaluations when possible. The selection of such patients is obviously an open issue.

\section{References}

1. S.A. Bahammam, M.M. Sharif, A.A. Jammah, A.S. Bahammam, Prevalence of thyroid disease in patients with obstructive sleep apnea. Respir. Med. 105, 1755-1760 (2011)

2. T. Bratel, A. Wennlund, K. Carlstrom, Pituitary reactivity, androgens and catecholamines in obstructive sleep apnea. Effects of continuous positive airway pressure treatment (CPAP). Respir. Med. 93, 1-7 (1999)

3. R.R. Grunstein, C.E. Sullivan, Sleep apnea and hypothyroidism: mechanisms and management. Am. J. Med. 85, 775-779 (1988)

4. R. Grunstein, J. Handelsman, S.J. Lawrence, C. Blackwell, J. Caterson, C.E. Sullivan, Neuroendocrine dysfunction in sleep apnea: reversal by continuous positive airways pressure therapy. J. Clin. Endocrinol. Metab. 68, 352-358 (1989)

5. F. Lanfranco, L. Gianotti, S. Pivetti, F. Navone, R. Rossetto, F. Tassone, V. Gai, E. Ghigo, M. Maccario, Obese patients with obstructive sleep apnoea syndrome show a peculiar alteration of the corticotroph but not of the thyrotroph and lactotroph function. Clin. Endocrinol. 60, 41-48 (2004)

6. F. Lanfranco, G. Motta, M.A. Minetto, M. Baldi, M. Balbo, E. Ghigo, E. Arvat, M. Maccario, Neuroendocrine alterations in obese patients with sleep apnea syndrome. Int. J. Endocrinol. 2010, 474518 (2010)

7. C.C. Lin, K.W. Tsan, P.J. Chen, The relationship between sleep apnea syndrome and hypothyroidism. Chest 102, 1663-1667 (1992)

8. N. Meston, R.J.O. Davies, R. Mullins, C. Jenkinson, J.A.H. Wass, J.R. Stradling, Endocrine effects of nasal continuous positive airway pressure in male patients with obstructive sleep apnoea. J. Intern. Med. 254, 447-454 (2003)

9. T. Mete, Y. Yalcin, D Berker, B. Ciftci, S. Guven Firat, O. Topaloglu, H. Cinar Yavuz, S. Guler, Relationship between obstructive sleep apnea syndrome and thyroid diseases. Endocrine (2013) [Epub ahead of print]

10. S.A. Mickelson, T. Lian, L. Rosenthal, Thyroid testing and thyroid hormone replacement in patients with sleep disordered breathing. Ear Nose Throat J. 78, 768-771, 774-775 (1999)

11. F. Rosenow, V. McCarthy, A.C. Caruso, Sleep apnoea in endocrine diseases. J. Sleep Res. 7, 3-11 (1998)

12. N.M. Skjodt, R. Atkar, P.A. Easton, Screening for hypothyroidism in sleep apnea. Am. J. Respir. Crit. Care Med. 160, 732-735 (1999) 